UT-854

RESCEU-20/99

UTAP-332

\title{
Initial condition for new inflation in supergravity
}

\author{
T. Asaka \\ Department of Physics, School of Science, University of Tokyo, Tokyo 113-0033, Japan \\ M. Kawasaki \\ Research Center for the Early Universe, School of Science, University of Tokyo, Tokyo 113-0033, \\ Japan \\ Masahide Yamaguchi \\ Department of Physics, School of Science, University of Tokyo, Tokyo 113-0033, Japan
}

(June 15 1999)

\begin{abstract}
We study the initial value problem of new inflation models in the scheme of supergravity. We find that an inflaton generally acquires a mass of the order of the Hubble parameter via gravitationally suppressed interactions with particles in the thermal bath of the universe. This additional mass term stabilizes dynamically the inflaton at the local maximum of the potential, which offers the initial condition of new inflation.
\end{abstract}

PACS: $98.80 . \mathrm{Cq}, 12.60 . \mathrm{Jv}$ 
Inflation [1] is very attractive because it solves the horizon and flatness problem of the standard big-bang cosmology and also gives the primordial density fluctuations observed by the Cosmic Background Explorer (COBE) satellite. In general inflation is caused by some scalar field called the "inflaton" which has a very flat potential. Initially the inflaton is assumed to be displaced from the true minimum of the potential and to give a nonzero vacuum energy. While the inflaton rolls down toward the true minimum very slowly, this vacuum energy dominates the energy of the universe and the exponentially expanding era, i.e., inflation, is realized.

Here, we would like to consider inflation models in the supersymmetric (SUSY) theories. The SUSY is the most attractive extension beyond the standard model of particle physics, since it stabilizes the electroweak scale against the radiative corrections and realizes the unification of the standard gauge couplings. Therefore, it is important to investigate inflation in the scheme of SUSY and also of its local version, i.e., supergravity.

So far, many SUSY inflation models have been proposed [2] and they are roughly classified to three types: chaotic [3], hybrid [4], and new [5] inflation. Within the framework of supergravity new inflation is favored because it naturally gives the solution to the gravitino problem. To avoid overproduction of gravitinos, the sufficiently low reheating temperature $T_{R} \lesssim 10^{8} \mathrm{GeV}$ is required [6]. Such a low reheating temperature is naturally offered in SUSY new inflation models [7] 9].

New inflation, however, suffers from a serious problem about the initial value of the inflaton $\phi$ [10]. Successful inflation requires the initial value be very close to the local maximum of the potential $(\phi \simeq 0)$ not the true minimum $(\phi=\langle\phi\rangle)$. But there is no reason to take such an initial condition over the horizon scale, because the potential of the inflaton is very flat to satisfy the slow roll conditions [10]. One might imagine that the inflaton is set to be $\phi=0$ due to the finite temperature effects via rapid interactions with particles in the thermal bath. However, such interactions generally break the flatness of the inflaton potential. Moreover, the inflaton usually receives large fluctuations of thr order of the cosmic temperature so that the required initial value of the inflaton is not realized over the horizon 
scale.

Izawa, Kawasaki, and Yanagida [11] found that another inflation (called preinflation) which takes place before new inflation solves the initial value problem. During preinflation the inflaton of new inflation acquires an additional mass term $\sim H_{I}^{2} \phi^{2}\left(H_{I}\right.$ is the Hubble constant during preinflation) through the supergravity effects. This effective mass is large enough for the inflaton to oscillate around $\phi=0$. Since the amplitude of the oscillation decreases at the rate $R^{-3 / 2}$ ( $R$ is the scale factor), the inflaton rapidly settles at $\phi=0$ if preinflation lasts long enough. However, this preinflation is introduced besides new inflation only to solve the initial value problem.

In this paper, we show that the inflaton, in general, gains the effective mass of the order of the Hubble parameter in the radiation-dominated universe through the gravitationally suppressed interactions with particles in the thermal bath. Therefore, the inflaton rolls down toward $\phi=0$ dynamically, which explains the initial condition of new inflation without introducing another inflation.

Let us consider the Kähler potential for the inflaton in the supergravity Lagrangian. In general, the Kähler potential for the supermultiplets of the inflaton $\phi$ of new inflation and the SUSY standard-model particles $\chi_{i}$ takes the form

$$
\begin{aligned}
K\left(\phi, \chi_{i}\right)= & |\phi|^{2}+\sum_{i}\left|\chi_{i}\right|^{2}+\frac{k}{4}|\phi|^{4}+\sum_{i} \frac{k_{i}}{4}\left|\chi_{i}\right|^{4} \\
& +\sum_{i} \lambda_{i}|\phi|^{2}\left|\chi_{i}\right|^{2}+\cdots
\end{aligned}
$$

where $k, k_{i}$, and $\lambda_{i}$ denote couplings of order unity and the ellipsis denotes higher order terms. Here and hereafter, we set the gravitational scale $M_{G} \simeq 2.4 \times 10^{18} \mathrm{GeV}$ equal to unity. The last term in Eq. (11) leads to the interaction Lagrangian

$$
\mathcal{L}_{\text {int }}=\sum_{i} \lambda_{i}|\phi|^{2} \partial_{\mu} \chi_{i}^{*} \partial^{\mu} \chi_{i}
$$

\footnotetext{
${ }^{1}$ Preinflations are also considered in a different context by Ref. [12].
} 
Here we use the same letters for the corresponding scalar components of the supermultiplets. Below the gravitational scale, these interactions decouple and the inflaton cannot be thermalized. On the other hand, the fields $\chi_{i}$ can be in thermal equilibrium, since they have some charges of the standard-model gauge groups and also have some Yukawa interactions.

We are now at the point to show how the interactions (2) stabilize the inflaton at $\phi \simeq 0$, which is required for the initial condition of new inflation. Just below the gravitational scale, the inflaton is assumed to be located at the true minimum of the potential $(\phi=\langle\phi\rangle)$. For temperature $T \simeq 10^{16}-10^{17} \mathrm{GeV}$, the interaction rates of $\chi_{i}$ via gauge (or Yukawa) interactions become larger than the expansion of the universe and they are in thermal equilibrium. After this epoch the inflaton receives an additional mass through the interactions (2) as follows: The thermal average of the operators $\partial_{\mu} \chi_{i}^{*} \partial^{\mu} \chi_{i}$ are estimated as

$$
\begin{aligned}
\left\langle\partial_{\mu} \chi_{i}^{*} \partial^{\mu} \chi_{i}\right\rangle & =-\left\langle\chi_{i}^{*} \partial_{\mu} \partial^{\mu} \chi_{i}\right\rangle \\
& =m_{\chi_{i}}^{2}(T)\left\langle\chi_{i}^{*} \chi_{i}\right\rangle=m_{\chi_{i}}^{2}(T) T^{2} / 12,
\end{aligned}
$$

where $m_{\chi_{i}}^{2}(T)$ are thermal masses squared for $\chi_{i}$ and given by $b_{\chi_{i}} T^{2}$ with $b_{\chi_{i}}$ being constants of order unity depending on the interactions of $\chi_{i}$. Therefore, the inflaton obtains the effective mass of order of the Hubble parameter as

$$
m_{\mathrm{eff}}^{2}=\sum_{i} \lambda_{i} b_{\chi_{i}} T^{4} / 12=c^{2} H^{2}
$$

since $T^{4} \sim H^{2}$ in the radiation dominated era. Here we assumed $\lambda_{i}$ are positive so that the effective mass squared becomes positive. Also, $c$ is a constant of order unity. This mass term modifies the evolution of the inflaton, which is described by the equation

$$
\ddot{\phi}+3 H \dot{\phi}+m_{\text {eff }}^{2} \phi=0
$$

and we find $\phi \propto R^{-1 / 2+\sqrt{1 / 4-c^{2}}}$ using $\dot{H}=-2 H^{2}$ in the radiation-dominated universe. Then, for $c>1 / 2$, we obtain

$$
\phi=\phi_{*}\left(\frac{R}{R_{*}}\right)^{-\frac{1}{2}} \cos \left[\sqrt{c^{2}-\frac{1}{4}} \ln \left(\frac{R}{R_{*}}\right)\right],
$$


where $\phi_{*}$ and $R_{*}$ are the amplitude and scale factor when the fields $\chi_{i}$ are in thermal equilibrium at temperature $T_{*}$. Thus, for $T<T_{*}$, the inflaton oscillates around $\phi=0$ with amplitude decreasing as $R^{-1 / 2}$ and it lasts until the vacuum energy $v^{4}$ of the inflaton becomes comparable to the radiation energy. Thus, the initial value of the inflaton $\phi_{I}$ when new inflation starts becomes at most $\left(v / T_{*}\right)^{1 / 2}\langle\phi\rangle$. If the factor $\left(v / T_{*}\right)^{1 / 2}$ is small enough, we can avoid the initial value problem. If Note that the energy density of the inflaton oscillation decreases as $R^{-5}$ so that the energy is dominated by the radiation until the start of new inflation, and also that the inflaton does not receive large fluctuations since the interactions (2) are suppressed by the gravitational scale.

The present mechanism for the dynamical tuning of the initial value of the inflaton applies for the generic new inflation models in supergravity [7 9]. As a concrete example, we consider a model proposed by Izawa and Yanagida [8] with the superpotential

$$
W=v^{2} \Phi-\frac{g}{n+1} \Phi^{n+1},
$$

where $v$ is the scale of the model, $g$ a coupling constant, and $n$ a positive integer with $n \geq 3$. Note that the coupling through the superpotential between the inflaton and the standard SUSY particles $\xi_{i}$ is prohibited by the $U(1)_{R}$ symmetry. From the Kähler potential (四) the inflaton $\phi=\sqrt{2}(\operatorname{Re} \Phi)$ obtains the potential as

$$
V(\phi)=v^{4}-\frac{1}{2} k v^{4} \phi^{2}-\frac{g}{2^{\frac{n}{2}-1}} v^{2} \phi^{n}+\frac{g^{2}}{2^{n}} \phi^{2 n},
$$

for $\phi<\langle\phi\rangle=\sqrt{2}\langle\Phi\rangle$. The true vacuum of the inflaton is estimated as

$$
\langle\Phi\rangle=\langle\phi\rangle / \sqrt{2}=\left(v^{2} / g\right)^{1 / n}
$$

\footnotetext{
${ }^{2}$ If we are allowed to tune the constant $c$, we can make the initial value $\phi_{I}$ much smaller than $\phi_{*}\left(v / T_{*}\right)^{1 / 2}$. From Eq. (6) $), \phi_{I}=0$ is achieved for $c=c_{0}$ given by $c_{0}^{2}-1 / 4=(\pi / 2+m \pi)^{2} / \ln ^{2}\left(T_{*} / v\right)$ where $m$ is an integer. Notice that due to the logarithmic dependence on $T_{*} / v$ of the period, $\phi_{I} \simeq 0$ is realized by mild tuning of $c\left(\simeq c_{0}\right)$.
} 
It has been shown in Ref. [8] that the slow-roll conditions for the inflaton are satisfied for $k<1$ and $\phi \lesssim \phi_{f}$ where

$$
\phi_{f} \simeq \sqrt{2}\left(\frac{(1-k) v^{2}}{g n(n-1)}\right)^{\frac{1}{n-2}}
$$

which provides the value of $\phi$ when inflation ends. The field value $\phi_{N}$, when the observable universe crossed the horizon during inflation, is given by

$$
\phi_{N} \simeq \sqrt{2}\left(\frac{v^{2} k}{n g}\right)^{\frac{1}{n-2}} \exp \left[-k\left(N+\frac{k n-1}{k(1-k)(n-2)}\right)\right],
$$

with $N \sim 50$. Also, the amplitude of the density fluctuations $\delta \rho / \rho$ is given by

$$
\frac{\delta \rho}{\rho} \simeq \frac{1}{5 \sqrt{3} \pi} \frac{V^{3 / 2}\left(\phi_{N}\right)}{\left|V^{\prime}\left(\phi_{N}\right)\right|} \simeq \frac{1}{5 \sqrt{3} \pi} \frac{v^{2}}{k \phi_{N}},
$$

and the spectral index $n_{s}$ of the density fluctuations is given by $n_{s} \simeq 1-2 k$. Since the COBE data [13] implies $n_{s}=1.0 \pm 0.2$, we should take $k \lesssim 0.1$, and the COBE normalization gives $v^{2} /\left(k \phi_{N}\right) \simeq 5.3 \times 10^{-4}$. For example, we consider the case of $n=6$ with $g=0.1$ and $k=0.02$. Then, we obtain $v \simeq 4.9 \times 10^{14} \mathrm{GeV},\langle\phi\rangle \simeq 2.9 \times 10^{17} \mathrm{GeV}$, and $\phi_{N} \simeq 9.6 \times 10^{15}$ $\mathrm{GeV}$. In the present scenario, the initial value of the inflaton $\phi_{I}$ is given by $\phi_{I} \lesssim\left(v / T_{*}\right)^{1 / 2}\langle\phi\rangle$ due to the additional mass (4T) so that it becomes smaller than $\phi_{N}$ if $T_{*} \gtrsim 1.6 \times 10^{17} \mathrm{GeV}$. [ Therefore, the existence of the nonrenormalizable interactions (2), fit which is inevitable in the framework of the supergravity, can naturally solve the initial value problem of new inflation.

Finally, we would like to remark on the flatness problem in the present model. Since the energy scale at the beginning of new inflation is much lower than the gravitational scale, the

${ }^{3}$ If one takes smaller $g$ or larger $n$, the required $T_{*}$ becomes smaller. If one takes larger $k$, the required $T_{*}$ becomes larger, but in this case the spectrum of the density fluctuations deviates from the scale invariant form too much.

${ }^{4}$ Through these interactions, the inflaton can decay into the SUSY standard-model particles and the universe reheats. In the present model, the reheating temperature $T_{\mathrm{RH}}$ becomes $T_{\mathrm{RH}} \sim 10^{6-8}$ $\mathrm{GeV}$, which is low enough to avoid the overproduction of gravitinos [6]. 
question may be raised why the universe lives so long (flatness problem). The preinflation, if it occurs near the gravitational scale, may avoid it [11]. However, this difficulty does not occur if the universe was created quantum mechanically as flat, or open.

This work was partially supported by the Japanese Society for the Promotion of Science (T.A.,M.Y.) and "Priority Area: Supersymmetry and Unified Theory of Elementary Particles(\#707)"(M.K.). 


\section{REFERENCES}

[1] A. H. Guth, Phys. Rev. D23, 347 (1981), K. Sato, Mon. Not. R. Astron. Soc. 195, 467 (1981).

[2] See, for a review, D. H. Lyth and A. Riotto, Phys. Rep. 314, 1 (1999).

[3] A. D. Linde, Phys. Lett. 129B, 177 (1983).

[4] A. D. Linde, Phys. Lett. B259, 38 (1991).

[5] A. D. Linde, Phys. Lett. 108B, 389 (1982), A. Albrecht and P. J. Steinhardt, Phys. Rev. Lett. 48, 1220 (1982).

[6] M. Yu. Khlopov and A.D. Linde, Phys. Lett. 138B, 265 (1984); J. Ellis, E. Kim and D.V. Nanopoulos, ibid, 145B, 181(1984); M. Kawasaki and T. Moroi, Prog. Theor. Phys. 93, 879 (1995).

[7] K. Kumekawa, T. Moroi, and T. Yanagida, Prog. Theor. Phys. 92, 437 (1994).

[8] K. I. Izawa and T. Yanagida, Phys. Lett. B393, 331 (1997).

[9] M. Dine and A. Riotto, Phys. Rev. Lett. 79, 2632 (1997).

[10] For example, A.D. Linde, Particle Physics and Inflationary Cosmology, (Harwood, Chur, Switzerland, 1990).

[11] K. I. Izawa, M. Kawasaki, and T. Yanagida, Phys. Lett. B411, 249 (1997).

[12] J. A. Adams, G. G. Ross, and S. Sarkar, Nucl. Phys. B503, 405 (1997); G. Lazarides and N. Tetradis, Phys. Rev. D58, 123502 (1998); C. Panagiotakopoulos and N. Tetradis, ibid. D59, 083502 (1999).

[13] C.L. Bennett et al., Astrophys. J. Lett. 464,L1 (1996). 